\title{
A Note On "Pythagorean Uncertain Linguistic Hesitant Fuzzy Weighted Averaging Operator and Its Application in Financial Group Decision Making"
}

\section{S.S. Appadoo ( $\sim$ SS.Appadoo@umanitoba.ca )}

University of Manitoba

Mohammadreza Makhan

University of Manitoba

Amit Kumar

Thapar Institute of Engineering and Technology

\section{Research Article}

Keywords: PULHFSs, Aggregation operators, MAGDM

Posted Date: January 24th, 2022

DOI: https://doi.org/10.21203/rs.3.rs-1248532/v1

License: (c) (i) This work is licensed under a Creative Commons Attribution 4.0 International License.

Read Full License 


\title{
A note on "Pythagorean uncertain linguistic hesitant fuzzy weighted averaging operator and its application in financial group decision making"
}

\author{
S.S. Appadoo ${ }^{\mathrm{a} 1}$, Mohammadreza Makhan ${ }^{\mathrm{a}}$, Amit Kumar ${ }^{\mathrm{b}}$ \\ aDepartment of Supply Chain Management, Asper School of Business, University of \\ Manitoba, Winnipeg, Canada \\ ${ }^{\mathrm{b}} \mathrm{Sch}$ ool of Mathematics, Thapar Institute of Engineering \& Technology (Deemed to be \\ University), Patiala-147004, Punjab, India
}

\begin{abstract}
Shakeel et al. (Soft Comput. 24 (2020)1585-1597) proposed the concept of a Pythagorean uncertain linguistic hesitant fuzzy set (PULHFS), some arithmetic operations of Pythagorean uncertain linguistic hesitant fuzzy sets (PULHFSs), an approach for comparing PULHFSs and a Pythagorean uncertain linguistic hesitant fuzzy weighted averaging (PULHFWA) operator as well as it extensions. Also, using the proposed comparing approach and the proposed aggregation operators, Shakeel et al. proposed a method for solving multiattribute group decision making (MAGDM) problems. In future other researchers may use Shakeel et al.'s work in their research work. However, it is observed that that the approach for comparing PULHFSs and aggregation operators, proposed by Shakeel et al. (2020), are not appropriate. Hence, the method for solving MAGDM problems, proposed by Shakeel et al., is also not appropriate. The aim of this note is to make the researchers aware about the inappropriateness of Shakeel et al.'s work. Furthermore, to point out that to resolve the inappropriateness of Shakeel et al.'s work (2020) is a challenging open research problem.
\end{abstract}

Keywords: PULHFSs, Aggregation operators, MAGDM

\section{Introduction}

Shakeel et al. (2020) proposed the concept of a PULHFS, some operational laws for

\footnotetext{
${ }^{1}$ Corresponding author

SS.Appadoo@umanitoba.ca
} 
PULHFSs, an approach for comparing PULHFSs and some aggregation operators for aggregating PULHFSs. Also, using the proposed comparing approach and proposed aggregation operators, Shakeel et al. (2020) proposed a method for solving such MAGDM problems in which the rating value of each alternative over an attribute is represented by a PULHFS.

In this note, it is shown that it is inappropriate to use Shakeel et al.'s approach (2020) for comparing PULHFSs as it fails to distinguish two distinct PULHFSs. Also, it is shown that as the monotonicity property is not satisfying for Shakeel et al.'s PULHFWA operator (2020). So, PULHFWA operator (2020) is not valid as an averaging operator is said to be valid only if it satisfies monotonicity property in addition to some other properties. Finally, it is pointed out that to resolve the inappropriateness of Shakeel et al.'s work (2020) may be considered as a challenging open research problem.

This note is organized as follows. In Section 2, Shakeel et al.'s approach (2020) for comparing PULHFSs is discussed. In Section 3, an example is considered to show that Shakeel et al.'s approach (2020) for comparing PULHFSs fails to distinguish two distinct PULHFSs. In Section 4, an example is considered to show that the monotonicity property is not satisfying for Shakeel et al.'s PULHFWA operator (2020). Hence, Shakeel et al.'s PULHFWA operator (2020) is not valid. Section 5 concludes the paper.

\section{Shakeel et al.'s approach for comparing PULHFSs}

Shakeel et al. (2020) proposed the following approach for comparing two PULHFSs $\tilde{p}_{1}=\left(\left[s_{\theta_{\left(p_{1}\right)}}, s_{t_{\left(p_{1}\right)}}\right],\left(\alpha_{\tilde{p}_{1}}, \beta_{\tilde{p}_{1}}\right)\right)$ and $\tilde{p}_{2}=\left(\left[s_{\theta_{\left(p_{2}\right)}}, s_{t_{\left(p_{2}\right)}}\right],\left(\alpha_{\tilde{p}_{2}}, \beta_{\tilde{p}_{2}}\right)\right)$ where,

(i) $\quad 0 \leq \alpha_{\tilde{p}_{i}} \leq 1,0 \leq \beta_{\tilde{p}_{i}} \leq 1, i=1,2$.

(ii) $\quad 0 \leq \alpha_{\tilde{p}_{i}}^{2}+\beta_{\tilde{p}_{i}}^{2} \leq 1, i=1,2$. 
(iii)

$$
s_{\theta_{\left(p_{i}\right)}}, s_{t_{\left(p_{i}\right)}} \in\left\{s_{0}, s_{1}, s_{2}, \ldots, s_{l-1}\right\}, i=1,2
$$

(iv) $\quad l$ is an odd positive integer.

Step 1: Calculate $S\left(\tilde{p}_{1}\right)$ and $S\left(\tilde{p}_{2}\right)$ and check that $S\left(\tilde{p}_{1}\right)<S\left(\tilde{p}_{2}\right)$ or $S\left(\tilde{p}_{1}\right)>S\left(\tilde{p}_{2}\right)$ or $S\left(\tilde{p}_{1}\right)=S\left(\tilde{p}_{2}\right)$

where,

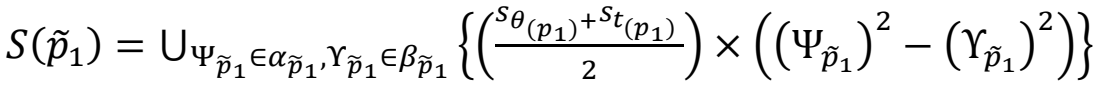

and

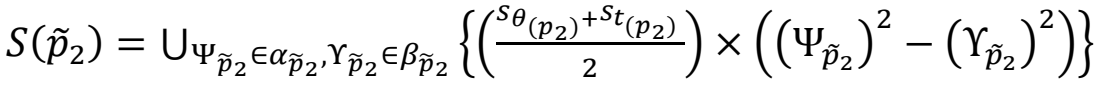

Case (i): If $S\left(\tilde{p}_{1}\right)<S\left(\tilde{p}_{2}\right)$, then $p_{1} \prec p_{2}$.

Case (ii): If $S\left(\tilde{p}_{1}\right)>S\left(\tilde{p}_{2}\right)$, then $p_{1}>p_{2}$.

Case (iii): If $\left(\tilde{p}_{1}\right)=S\left(\tilde{p}_{2}\right)$, then go to Step 2 .

Step 2: Calculate $h\left(\tilde{p}_{1}\right)$ and $h\left(\tilde{p}_{2}\right)$ and check that $h\left(\tilde{p}_{1}\right)<h\left(\tilde{p}_{2}\right)$ or $h\left(\tilde{p}_{1}\right)>h\left(\tilde{p}_{2}\right)$ or $h\left(\tilde{p}_{1}\right)=h\left(\tilde{p}_{2}\right)$

where,

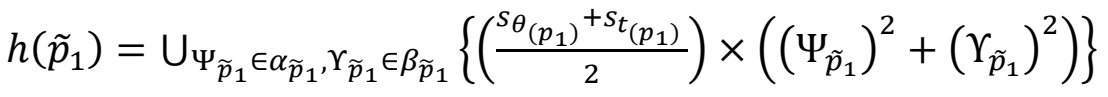

and

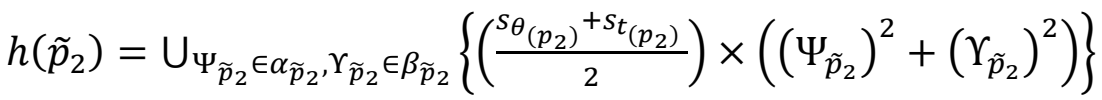

Case (i): If $h\left(\tilde{p}_{1}\right)<h\left(\tilde{p}_{2}\right)$, then $\tilde{p}_{1} \prec \tilde{p}_{2}$.

Case (ii): If $h\left(\tilde{p}_{1}\right)>h\left(\tilde{p}_{2}\right)$, then $\tilde{p}_{1}>\tilde{p}_{2}$.

Case (iii): If $h\left(\tilde{p}_{1}\right)=h\left(\tilde{p}_{2}\right)$, then $\tilde{p}_{1}=\tilde{p}_{2}$.

\section{Inappropriateness of Shakeel et al.'s approach for comparing two PULHFSs}

Let $\tilde{p}_{1}=\left(\left[s_{1}, s_{2}\right],(0.6,0.5)\right)$ and $\tilde{p}_{2}=\left(\left[s_{0}, s_{3}\right],(0.6,0.5)\right)$ be two PULHFSs. It is obvious that $\tilde{p}_{1}$ is not equal to $\tilde{p}_{1}$. While, in this section, it is shown that according to the 
Shakeel et al.'s approach (2020) for comparing PULHFSs, discussed in Section 2, $\tilde{p}_{1}=\tilde{p}_{2}$. Hence, the Shakeel et al.'s approach (2020) for comparing PULHFSs, discussed in Section 2, is not valid.

Step 1: According to Step 1 of Shakeel et al's. approach (2020), discussed in Section 2,

$S\left(\tilde{p}_{1}\right)=\frac{1+2}{2} \times\left(0.6^{2}-0.5^{2}\right)=0.165$ and $\left(\tilde{p}_{2}\right)=\frac{0+3}{2} \times\left(0.6^{2}-0.5^{2}\right)=0.165$.

Step 2: Since $S\left(\tilde{p}_{1}\right)=S\left(\tilde{p}_{2}\right)$, so according to Case (iii) of Step 1 of Shakeel et. al's approach (2020), discussed in Section 2, there is a need to calculate $h\left(p_{1}\right)$ and $h\left(p_{2}\right)$.

$h\left(\tilde{p}_{1}\right)=\frac{1+2}{2} \times\left(0.6^{2}+0.5^{2}\right)=0.915$ and $h\left(\tilde{p}_{2}\right)=\frac{1+2}{2} \times\left(0.6^{2}+0.5^{2}\right)=0.915$.

Since, $h\left(\tilde{p}_{1}\right)=h\left(\tilde{p}_{2}\right)$, so according to the Case (iii) of the Step 2 of Shakeel et al.'s approach (2020), discussed in Section 2, $\tilde{p}_{1}=\tilde{p}_{2}$. While in the actual case $\tilde{p}_{1} \neq \tilde{p}_{2}$, hence Shakeel et al.'s approach (2020), discussed in Section 2, is not valid.

\section{Invalidity of Shakeel et al.'s PULHFWA operator}

Shakeel et al. (2020) proposed the following PULHFWA operator to aggregate $m$

$$
\text { PULHFSs } \tilde{p}_{i}=\left(\left[s_{\theta_{\left(p_{i}\right)}}, s_{t_{\left(p_{i}\right)}}\right],\left(\alpha_{\tilde{p}_{i}}, \beta_{\tilde{p}_{i}}\right)\right) ; i=1,2, \ldots, m \text {, }
$$

$\operatorname{PULHFWA}\left(p_{1}, p_{2}, \ldots, p_{m}\right)=$

$\left(\left[\sum_{i=i}^{m}\left(s_{w_{i} \theta_{\left(p_{i}\right)}}\right), \sum_{i=1}^{m}\left(s_{w_{i} t_{\left(p_{i}\right)}}\right)\right], \cup_{\Psi_{\widetilde{p}_{i}} \in \alpha_{\tilde{p}_{i}}} \sqrt{\left(1-\prod_{i=1}^{m}\left(1-\Psi_{\tilde{p}_{i}}{ }^{2}\right)^{w_{i}}\right)}, \cup_{\Upsilon_{\widetilde{p}_{i} \in \tilde{p}_{i}}}\left(\prod_{i=1}^{m} \Upsilon_{\tilde{p}_{i}}{ }^{w_{i}}\right)\right)$

where,

$0 \leq w_{i} \leq 1$ and $\sum_{i=1}^{m} w_{i}=1$

To show the validity of the proposed PULHFWA operator, Shakeel et al. (2020) discussed some necessary properties including monotonicity property which is defined as follows:

$$
p_{i} \preccurlyeq p_{i}^{*} ; i=1,2, \ldots, m \Longrightarrow \operatorname{PULHFWA}\left(p_{1}, p_{2}, \ldots, p_{m}\right) \preccurlyeq \operatorname{PULHFWA}\left(p^{*}{ }_{1}, p^{*}{ }_{2}, \ldots, p^{*}{ }_{m}\right)
$$


In this section, an example is considered to show that the monotonicity property is not satisfying for Shakeel et al.'s PULHFWA operator (2020). Hence, Shakeel et al.'s PULHFWA operator (2020) is not valid as an averaging operator is said to be valid only if it satisfies some properties including the monotonicity property.

Let $\tilde{p}_{1}=\left(\left[s_{0}, s_{6}\right],(0.6,0.2)\right), \tilde{p}_{2}=\left(\left[s_{0}, s_{6}\right],(0.9,0.2)\right)$ and $\tilde{p}_{3}=\left(\left[s_{6}, s_{2}\right],(1,0)\right)$ be three PULHFSs. Also, let $=7, w_{1}=0.9$ and $w_{2}=0.1$. Then,

$S\left(\tilde{p}_{1}\right)=\frac{0+6}{2} \times\left(0.6^{2}-0.2^{2}\right)=0.96$

$S\left(\tilde{p}_{2}\right)=\frac{0+6}{2} \times\left(0.9^{2}-0.2^{2}\right)=2.31$

Since, $S\left(\tilde{p}_{1}\right)<S\left(\tilde{p}_{2}\right) \quad$ So, according to Shakeel et al.'s approach (2020), discussed in Section $2, \tilde{p}_{1} \prec \tilde{p}_{2}$.

Now as $\tilde{p}_{1} \prec \tilde{p}_{2}$ and $\tilde{p}_{3}=\tilde{p}_{3}$. So, according to the monotonicity property, the relation PULHFWA $\left(\tilde{p}_{1}, \tilde{p}_{2}\right) \prec P U L H F W A\left(\tilde{p}_{2}, \tilde{p}_{3}\right)$ should be satisfied.

While, the following clearly indicates that $\operatorname{PULHFWA}\left(\tilde{p}_{1}, \tilde{p}_{3}\right)=\operatorname{PULHFWA}\left(\tilde{p}_{2}, \tilde{p}_{3}\right)$. Hence, the monotonicity property is not satisfying for Shakeel et al.'s PULHFWA operator (2020).

$\operatorname{PULHFWA}\left(\tilde{p}_{1}, \tilde{p}_{3}\right)=$ $\left(\left[s_{6 \times 0.1+0 \times 0.1}, s_{6 \times 0.9+2 \times 0.1}\right],\left(\sqrt{\left(1-\left(1-0.6^{2}\right)^{0.9} \times\left(1-1^{2}\right)^{0.1}\right)}, 0.2^{0.9} \times 0^{0.1}\right)\right)=$ $\left(\left[s_{0.6}, s_{5.6}\right],(1,0)\right)$

and

$\operatorname{PULHFWA}\left(\tilde{p}_{2}, \tilde{p}_{3}\right)=$ $\left(\left[s_{6 \times 0.1+0 \times 0.1}, s_{6 \times 0.9+2 \times 0.1}\right],\left(\sqrt{\left(1-\left(1-0.9^{2}\right)^{0.9} \times\left(1-1^{2}\right)^{0.1}\right)}, 0.2^{0.9} \times 0^{0.1}\right)\right)=$ $\left(\left[s_{0.6}, s_{5.6}\right],(1,0)\right)$.

\section{Conclusion}

In this note, it is pointed out that the approaches for comparing PULHFSs and the 
PULHFWA operator, proposed by Shakeel et al. (2020), is not valid. It is pertinent to mention that Shakeel et al.'s approach (2020) for solving PULHFSs based MAGDM problems is also not valid as the approach for comparing PULHFSs as well as the PULHFWA operator, proposed by Shakeel et al. (2020), are used in this approach. To resolve the inappropriateness of Shakeel et al.'s work (2020) may be considered as a challenging open research problem.

\section{Compliance with ethical standards}

Conflict of interest Authors declare that they have no conflict of interest.

\section{Acknowledgments}

The authors would like to thank Associate Editor "Professor Raffaele DS Cerulli" and anonymous reviewers for their insightful comments and suggestions.

\section{References}

Shakeel M, Shahzad M, Abdullah S (2020) Pythagorean uncertain linguistic hesitant fuzzy weighted averaging operator and its application in financial group decision making. Soft Comput. 24(3):1585-97 